\title{
Chosen aspects of thermal image processing - a new software for medical applications
}

\author{
by M. Więcek ${ }^{1}$, R. Strąkowski ${ }^{1}$, T. Jakubowska ${ }^{2}$, B. Więcek ${ }^{1}$
}

${ }^{1}$ Technical University of Łódź, Institute of Electronics

${ }^{2}$ Laser Diagnostic and Therapy Center at Technical University of Łódź

\section{Abstract}

In this paper, the useness of 2D wavelet transform of thermal image processing is presented for medical applications. The new software implementing in Matlab® is described. Thermal signature calculations based on first and second order statistical parameters are implemented. The paper presents the image features sensitivity on noise level and size of investigated object. The chosen results are presented.

\section{Introduction}

A growing interest of thermovision techniques in medicine stimulates the new developments in software engineering for medical applications. Among many imaging techniques, 2D Wavelet Transformation (WT) is actually used in many domains, such as telecommunication and signal processing for compression and extracting quantitative features from a signal. WT can be employed to get new features, representing both global and detail information. It is based on filtering of an image represented by rows and columns using both low and high pass linear filters (Fig. 1). After filtering, decimation is made to reduce number of pixels. The procedure can be repeated until low resolution subimages are obtained. Practically, the processing is stopped earlier, after 2-4 steps, and then the features are calculated from the filtered subimages [9-11].

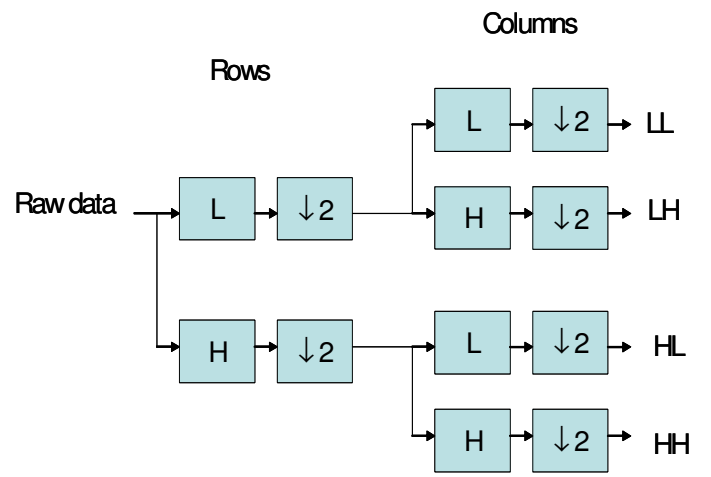

Fig. 1. $2 D$ wavelet transformation of an image

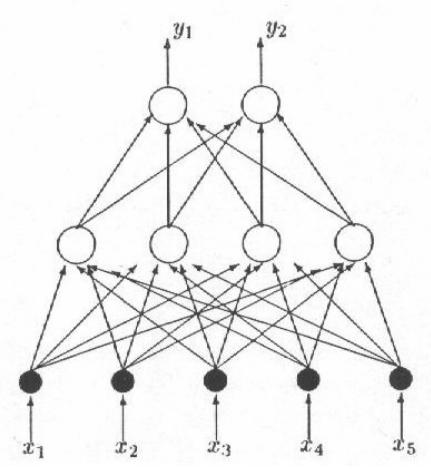

Fig. 2. Neural network example with input, single hidden and output layers

Having image features, an artificial neural network (ANN) may be used as an effective tool for image classification (Fig 2). The selected image features can be used as inputs. It means that the number of inputs nodes of the neural network is equal to the number of features using in the classification. ANN can have userdefined next hidden layers which allow additional nonlinear processing of input features. Number of neuron in the first hidden layer can be equal or lower than the number of input ones, as shown in Fig. 2. As ANN is the nonlinear system, such technique allows the additional decorrelating and data reduction, what finally improves the classification. Such approach is known as Nonlinear Discriminant Analysis (NDA) [10,11]. It is well known that the training of ANN is a very important step in the entire protocol. It is a multivariable optimization problem typically based on back-propagation technique [8]. One needs enough data during learning phase to obtain the values of neuron weight coefficients. The effectiveness of image classification directly depends on quality of ANN learning process.

\section{New software for thermal image processing}

In order to verify the research assumptions, novel software was created in MATLAB environment (Fig.3). In Laser Diagnostic and Therapy Center, at Technical University of Lodz, the laboratory for parallel diagnosis of breast diseases using mammography, ultrasonography and thermography, was created. In the same place and in the same time, a patient can take the diagnosis using thermography, digital mammography, and ultrasonography. In our laboratory, a screening program has just been started, so we hope to collect enough images for ANN learning process. The software which is discussed in the paper is suitable for feature extracting and image classification either for X-ray, acoustic or thermal images.

There are many different methods allowing image features calculations. A large group of methods is based on statistical parameters. In the software that has been realized by the research group in the Institute of 
Electronics at Technical University of Łódź, first and second order statistical parameters are calculated. The parameters like mean value, standard deviation, skewness, kurtosis, etc. can be used to compare thermal images $[11,12]$. The first order statistical parameters methods use image's histogram to compute signatures, while the second order statistical parameters are defined for so-called co-occurrence matrix of the image $[8,9,11]$.

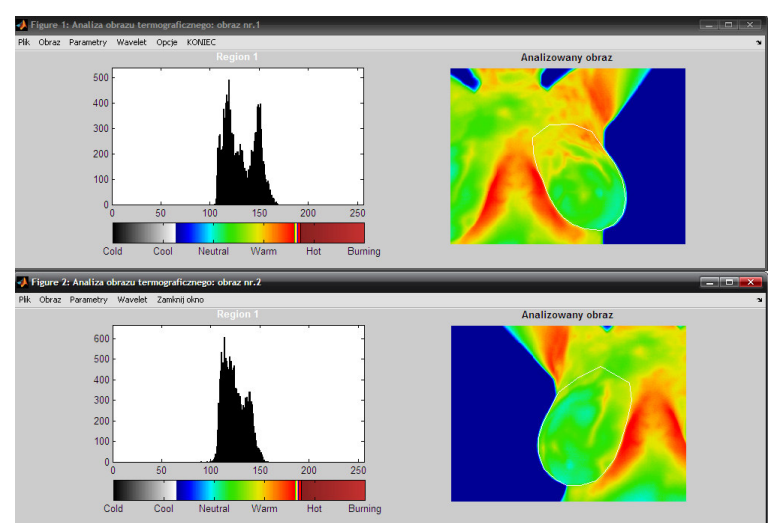

Fig. 3. Histogram and calculated features of a thermal image

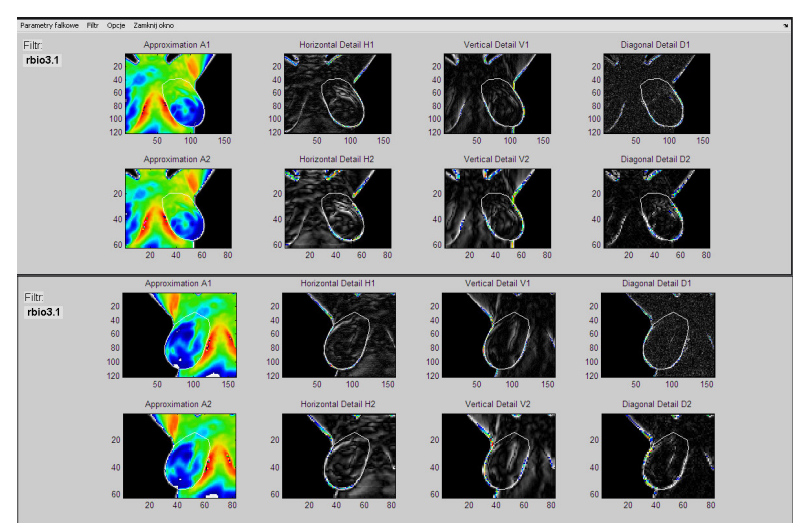

Fig. 4. Subimages obtained by $2 D$ wavelet transformation

In medical applications, one of the principal features of the thermal image is its symmetry of temperature patterns. Thermal images are usually asymmetrical in pathological cases. Any significant asymmetry can indicate a physiologic abnormality. This may be a pathological case (including cancer, fibrocystic disease, an infection or a vascular disease) or it might indicate an anatomical variant $[1,2,7,11,12]$.

The next group of methods implemented in the software is based on image transformations, such as linear filtering, Fourier or wavelet analysis. All these methods allow to regenerate an image which is processed or converted, and signatures are defined in different domain, eg.: frequency or scale. Well-known Karhunen-Loeve transformation is implemented as Principle Component Analysis (PCA). PCA is a technique that is usually used for reducing the dimensionality of multivariate data, preserving most of the variance, and decorrelating the parameters for better image classification [3, 4, 11]. Features' preprocessing which generates new parameters after linear or nonlinear transformations can be the next step in the procedure. It allows to get less correlated and lower order data. Two approaches are used, i.e.: PCA (Principal Component Analysis) and LDA (Linear Discriminant Analysis) [4, 6, 8]. Finally, classification can be performed using different Artificial Neural Networks (ANN), with or without additional hidden layers, and with different number of neurons. Alternatively, Nearest Neighbor Classification (NNC) can also be employed for such image processing.

\section{Chosen parameters sensitivity to noise level and scaling}

There are tens of thermal signatures that can be easily calculated using the procedures described above. One of the problems in choosing the right thermal parameters is their dependency from the noise and size of the image taken to the investigations. Noise strongly depends from the type of the camera used in measurements. This issue seems to be important as we have today two main thermovision camera types on the market microbolometr, uncooled and photon, cooled ones. Obviously, uncooled, cost-effective cameras have the thermal resolution significantly lower, reaching the level $40-50 \mathrm{mK}$ in contract to $15-20 \mathrm{mK}$ for cooled ones. The practical question can be posed, if there is a way to compensate the lower performance of the equipment by choosing the thermal signature less dependent from noise. In addition, because of the need of standardization of taking thermal images [11], it is necessary to estimate the right distance in between the camera and the subject. Definitely, the closer the patient, the more accurate is the thermal imaging, but due to practical reason, it is very difficult to keep the constant distance in time, during massive screening. For this reason the sensitivity of the parameters as a function of distance and size of the subject should be as low as possible. In addition, to compare the results obtained using different cameras, with different optics, choosing the less dependent thermal index from no. of pixels in the considered region of interest $(\mathrm{ROI})$ is recommended.

In order to find the less dependent thermal parameters from the level of additive noise and the size of ROI, the preliminary research was been performed. First and second order statistical parameters were considered [8,11]. In addition, 2D WTwas applied to calculate both histogram and co-occurrence matrix-based thermal signatures. A hypothesis was assumed that WT which is based on filtering (Fig. 1), should reduce the noise influence on the value of chosen parameters. Additionally, the considered parameter should allow differentiating the pathological and physiological cases in acceptable wide range of variation of the noise and size.

The investigation was carried out for thermal images of breast tumors and faces during physical exercises (Fig. 5, 7). In both cases, the wavelet transformation with biorthogonal filters was used. Then, the different thermal parameters were taken into account, and the best results were obtained for the second order statistical ones [11]. In order to differentiate healthy and pathological cases for breast investigation, 2nd order variance and correlation were chosen (Fig. 6). For an image of a man's face before and after the exercises the variance calculated from the histogram (1st order) and co-occurrence matrix (2nd order) were the best and less depended from the noise 
and size of the image (Fig. 8). During the investigation, uniformly distributed noise with an amplitude An was added to each pixel intensity having the range of variation As. The noise level was defined as ratio An/As. The maximum noise level equal $25 \%$ denotes the noise amplitude is one quarter of the pixel dynamics, what is high enough for simulating the different camera performance. Size of the image is defined in the range 100-40\%. $100 \%$ means that the image is of full scale as it is presented in Fig.5 and Fig. 7. For maximally sized image, it has the area equal only to $40 \%$ of the full size object.
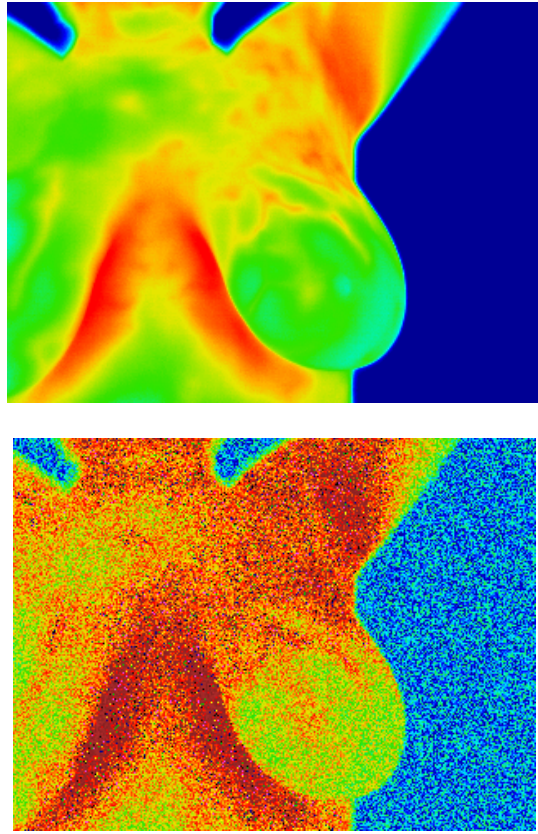
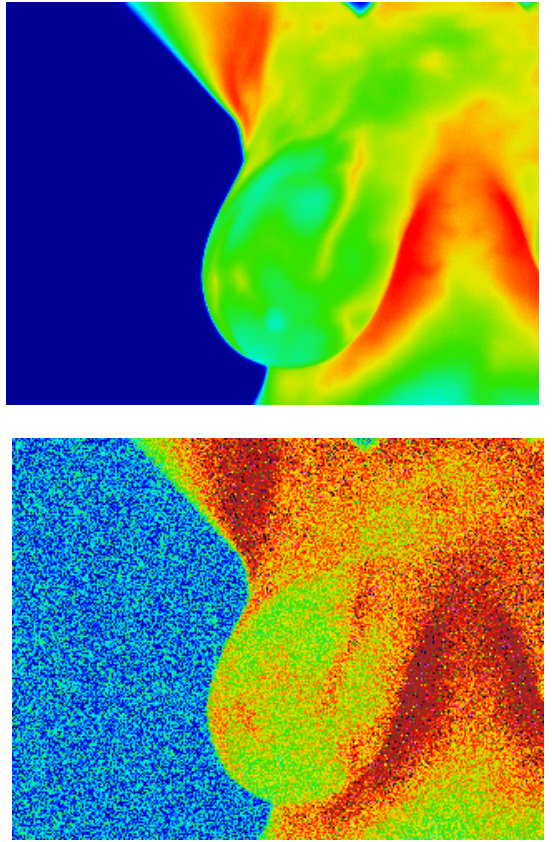

Fig. 5. Breast thermal images with $25 \%$ of additive noise, pathological (left) and healthy (right) cases - full size images
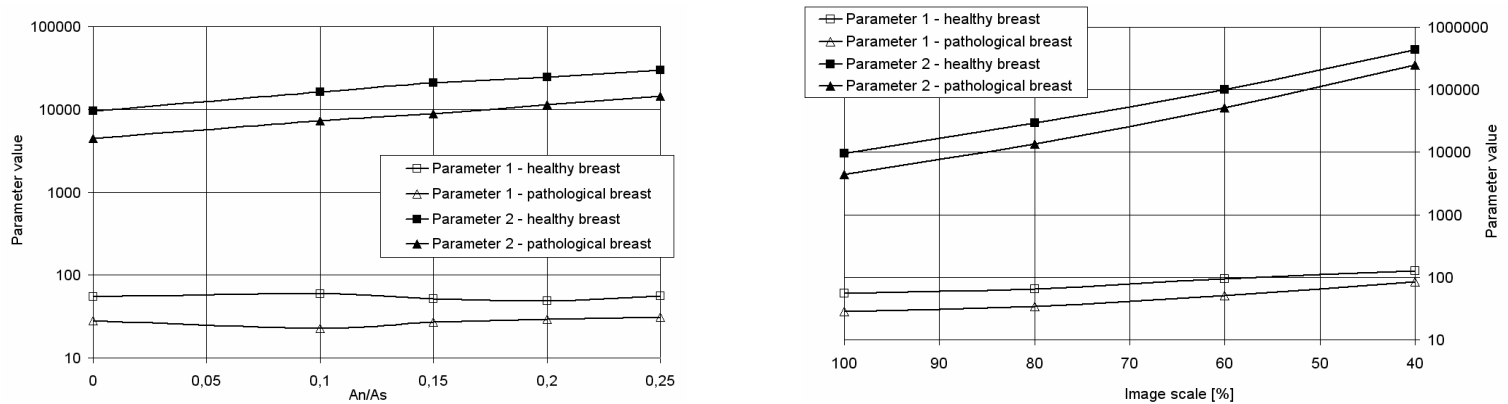

Fig. 6. 2nd order variance (parameter 1) and correlation (parameter 2) chosen for breast investigation, parameter values versus noise level (left), and image scale (right)

The preliminary investigations confirmed the expectation that among many different thermal signatures, the parameters calculated after 2D wavelet transformation using 2nd order statistical parameters are the most promising the screening purposes. Definitely, this investigation proved the necessity of selecting the best parameters. Different criteria have to be considered for such a selection. In this research the criteria of parameters' dependency from the noise and size of the image was verified. Obviously, discriminating healthy and pathological images has to be the main criterion. This investigation proved that such an approach can be the powerful tool for the medical diagnosis, and should be implemented in the software as an automatic or semiautomatic procedure.

\section{Conclusion}

Novel software for thermal image processing for medical applications is presented. This packet implements different method for image feature calculations, based 1st and 2nd order statistical parameters. In addition, chosen 2D transformations are used to get another set of thermal signatures. After image features' selection, a classification procedures are applies for image separating to differentiate the healthy and pathological cases during the screening protocols. Thermal image classification is a powerful and objective tool for many medical diagnostic protocols, e.g.: during breast tumor screening [8, 10-11]. 

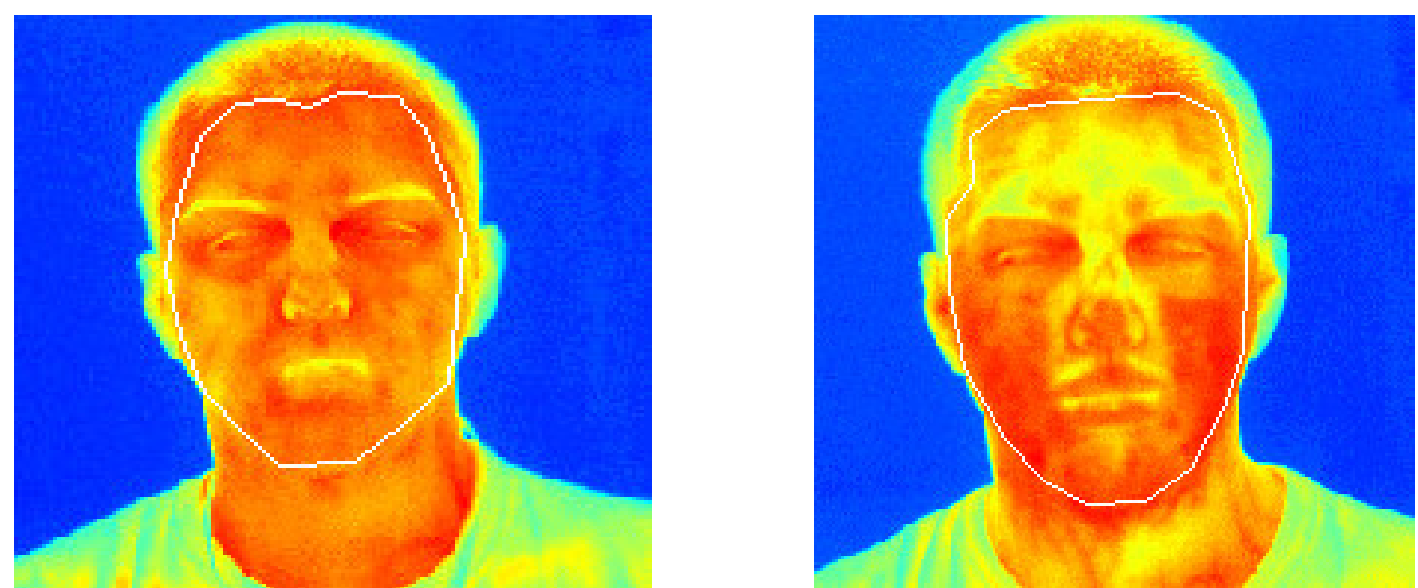

Fig. 7. Face's thermal images before (left) and after (right) physical exercises - full size images, different areas of ROls
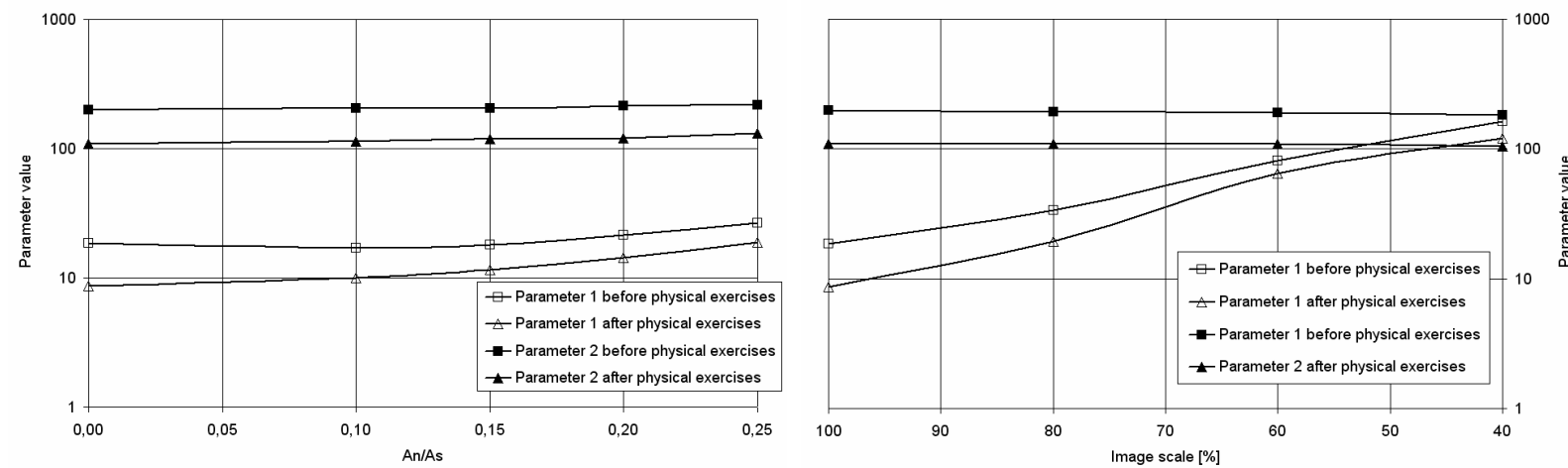

Fig. 8. 1st (parameter 1) and 2nd (parameter 2) order variance chosen for differentiating a face before and after exercises versus noise level (left), and image scale (right)

\section{REFERENCES}

[1] E.Y.K. Ng, L.N. Ung, F.C. Ng, L.S.J. Sim "Statistical Analysis Of Healthy And Malignant Breast Thermography", Journal of Medical Engineering \& Technology, Vol. 25 No 6 (Nov/Dec 2001) p.253-263./

[2] B. Wiecek, S. Zwolenik, "Thermal wave method - limits and potentialities of active thermography in biology and medicine", 2nd Joint EMBS-BMES Conference, 24th Annual International Conference of the IEEE Engineering in Medicine and Biology Society, BMES-EMS 2002, Huston, 23-26 Oct. 2002.

[3] I. T. Jolliffe, "Principal Component Analysis". New York, Springer-Verlag, 1986.

[4] B. F. J Manly, "Multivariate Statistical Method: A Primer". London, Chapman \& Hall, 1994.

[5] D. R. Causton, "A Biologist's Advanced Mathematics", London, Allen and Unwin, 1987.

[6] A. Materka, M. Strzelecki, R. Lerski, L. Schad, „Evaluation of Texture Features of Test Objects for Magnetic Resonance Imaging", Infotech Oulu Workshop on Texture Analysis in Machine Vision, June , 1999, Oulu, Finland.

[7] Michael Bennett "Breast Cancer Screening Using High-Resolution Digital Thermography", Total Health, Vol. 22 No 6 p.44, 1985.

[8] P. Debiec, M. Strzelecki, A. Materka, Evaluation of texture Generation Methods Based on CNN and GMRF Image Texture Models, Proc. of Int. Conf. on Signals and Electronic Systems, 17-20 October 2000, Ustron, pp. 187-192.

[9] M. Kociolek, A. Materka, M. Strzelecki, P. Szczypinski, "Discrete Wavelet Transform-Derived Features for Digital Image Texture Analysis", Proc. International Conference on Signals \& Electronic Systems ICSES'2001, Lodz, 18-21 September 2001, pp. 111-116

[10] P. Debiec, M. Strzelecki, A. Materka, "Evaluation of Texture Generation Methods Based on CNN and GMRF Image Texture Models", International Conference on Signals and Electronic Systems ICSES'2000, Ustron, Oct. 2000, pp. 187-192.

[11] Jakubowska, T., Wiecek, B., Wysocki, M.,Drews-Peszynski, C. "Thermal Signatures for Breast Cancer Screening Comparative Study, Proc. IEEE EMBS Conf. Cancun, Mexico, Sep 17-21, 2003.

[12] Amalu W.C., Hobbins W.B., Head F.J., Elliot R.L., Section III, chapt. 25. "Infrared Imaging of the Breast - An Overview" in "Medical Devices and Systems" Ed. Joseph D. Bronzino. CRC Press Taylor and Francis Group, 2006, s. 25-1 - 25-21. 